\title{
Applicability of CAAT Box-derived Polymorphism (CBDP) Markers for Analysis of Genetic Diversity in Durum Wheat
}

\author{
A. Etminan ${ }^{1 *}$, A. Pour-Aboughadareh ${ }^{2}$, R. Mohammadi $^{3}$, A. Noori ${ }^{1}$ and A. Ahmadi-Rad ${ }^{4}$ \\ ${ }^{1}$ Department of Biotechnology and Plant Breeding, College of Agriculture, Kermanshah Branch, \\ Islamic Azad University, Kermanshah, Iran \\ ${ }^{2}$ Department of Crop production and Breeding, Imam Khomeini International University, Qazvin, Iran \\ ${ }^{3}$ Dryland Agricultural Research Institute, Sararood branch, Agriculture Research, Education and Extension \\ Organization (AREEO), Kermanshah, Iran \\ ${ }^{4}$ Young Researchers and Elite Club, Kermanshah Branch, Islamic Azad University, Kermanshah, Iran
}

(Received 21 March 2017; Accepted 30 May 2017

Communicated by A. Börner)

\begin{abstract}
Progress in plant molecular tools has been resulted in the development of gene-targeted and functional marker systems. CAAT box region is a different pattern of nucleotides with a consensus sequence, GGCCAATCT, which situated upstream of the start codon of eukaryote genes and plays an important role during transcription. In the present study, several CAAT box-derived polymorphism (CBDP) primers were used for fingerprinting in mini-core collection of durum wheat (including internationally developed breeding lines and Iranian landraces). Twelve selected primers amplified 98 loci, of which all were polymorphic. The average values of the polymorphism information content $(P I C)$ and resolving power $(R p)$ were 0.31 and 9.16 , respectively, indicating a high level of variability among studied genotypes. Analysis of molecular variance (AMOVA) indicated that $92 \%$ of the total variation resided among populations. The values of the percentage polymorphic bands $(P P L)$, the observed $(\mathrm{Na})$ and effective ( $\mathrm{Ne}$ ) number of alleles, Nei's gene diversity $(\mathrm{He})$ and Shannon's information index $(I)$ for Iranian landraces were higher than the breeding lines. The Fandendrogram obtained by cluster analysis grouped all individuals into three main clusters. Our results showed a remarkable level of genetic diversity among studied durum wheat, especially among Iranian landraces, which can be interest for future breeding programs. More importantly, the present study also revealed that CBDP technique was efficient and powerful tool to assess genetic diversity in wheat germplasm. Hence, this technique could be employed individually or in combination with other molecular markers to evaluate genetic diversity and relations among different species.
\end{abstract}

Keywords: durum wheat, genetic variability, CBDP, Iranian landraces

\section{Introduction}

Wheat (common and durum) is one of the most important cereal crops with high economic value. Durum wheat (Triticum turgidum L. subsp. durum), as one of the major cereal crops, is the only tetraploid species of commercial wheat that widely cultivated in

*Corresponding author; E-mails: alietminan55@yahoo.com 
Mediterranean climates (Poursiahbidi et al. 2013). Genetic erosion in cultivated wheat suggests research for measuring genetic diversity in its germplasm. It is assumed that assessment of genetic variability is very important to maximize the efficiency of breeding programs (Pour-Aboughadareh et al. 2017). Hence, the knowledge of the extent and pattern of genetic diversity in the crop plants increases germplasm management and utilization.

In comparison to traditional methods, DNA fingerprinting provides a direct measure of variability at genetic levels for the evaluation of genetic diversity (Alikhani et al. 2014). In recent years, advances in the genomic tools have provided novel marker systems such as, functional and gene-targeted as well as the development of novel DNA-based marker systems. CAAT box-derived polymorphism (CBDP) is one of the novel, simple, and reliable gene-targeted marker systems. This technique activates the CAAT box region of promoters in plant genes (Singh et al. 2014). CAAT box has a different pattern of nucleotides with a consensus sequence, GGCCAATCT, situated $>80$ bp upstream of the start codon of eukaryote genes and plays an important role during transcription (Benoist et al. 1980). Furthermore, CBDP technique due to longer primers with higher annealing temperature will be more reliable and reproducible that random markers like as RAPDs or DAFs (Collard and Mackill 2009). Thus, this technique may have advantages over random markers for applications in QTL mapping and DNA fingerprinting (Andersen and Lubberstedt 2003). The use of molecular markers that easily and efficiently determine the level of genetic diversity is very important in the evaluation of the plant core collections. Previously, genetic diversity among durum wheat populations has been studied using a large number of morphological and molecular markers (Mardi et al. 2011; Poursiahbidi et al. 2012; Alsaleh et al. 2016; Etminan et al. 2016). The main goals of this work were: (i) to determine the genetic diversity using CBDP marker system with emphasis on Iranian landraces and (ii) to determine the potential of this technique for diagnostic fingerprinting of durum wheat genotypes.

\section{Materials and Methods}

\section{Plant materials and DNA extraction}

Fifty durum wheat (Triticum turgidum L. subsp. durum) genotypes including 21 landrace genotypes sampled from different location of Iran (population 1) and 29 improved genotypes provided by International Centre for Agricultural Research in the Dry Areas (ICARDA) (population 2) were considered for evaluation of genetic diversity using CBDP technique. Detailed information for these materials is listed in Table S1*. After seed germination and growth, the total genomic DNA was isolated from the fresh leaves of glasshousegrown plants according to the CTAB protocol (Doyle and Doyle 1987), and DNA quality was analyzed by $0.8 \%$ agarose gel electrophorese.

\footnotetext{
*Further details about the Electronic Supplementary Material (ESM) can be found at the end of the article.
} 


\section{CBDP-PCR amplification}

Twelve CBDP primer sequences, which used in the current study were designed according to Singh et al. (2014). The total reaction volume was $20 \mu \mathrm{L}$ containing $10 \mu \mathrm{L}$ master mix 2XPCR (ready-to-use PCR master mix 2X), $2 \mu \mathrm{L}$ of isolated DNA from each acces-

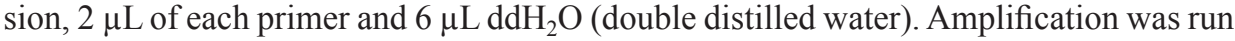
at $95^{\circ} \mathrm{C}$ for $4 \mathrm{~min}$, followed by 30 cycles of denaturation at $94{ }^{\circ} \mathrm{C}$ for 60 seconds, primer annealing at $50{ }^{\circ} \mathrm{C}$ for 60 seconds and primer elongation at $72{ }^{\circ} \mathrm{C}$ for 120 seconds. The final extension was $7 \mathrm{~min}$ at $72{ }^{\circ} \mathrm{C}$. The amplification reaction products were detected by $1.2 \%$ denaturing agarose gels stained with safe view II.

\section{Data analysis}

Firstly, amplification products were scored as absent (0) and present (1), compiling the data as a binary matrix. For comparing the banding patterns of primers, various genetic parameters such as, polymorphism information content $(P I C)$, resolving power $(R p)$ and marker index $(M I)$ were calculated. The distribution of genetic variation within and among populations (AMOVA) was estimated using GenALEx software (Peakall and Smouse 2006). The genetic variability indices, viz. the percentage of polymorphic loci $(P P L)$, the observed $(\mathrm{Na})$ and effective numbers of alleles $(\mathrm{Ne})$, Nei's gene diversity $(\mathrm{He})$, and Shannon's information index $(I)$ were estimated using POP-GENE software (Yeh et al. 1997). The Jaccard's (Jaccard 1908) genetic similarities matrix from the calculative data was used to construct a Fan-dendrogram using MEGA5 software (Tamura et al. 2011).

\section{Results}

Twelve CBDP primers generated a total of 98 scorable bands, of which all were polymorphic, accounting for $100 \%$. The total number of bands per primer ranged from 5 (by CBDP-13 and CBDP-15) to 12 (by CBDP-1), with an average of 8.17 bands per primer. The PIC values varied from 0.14 (by CBDP-14) to 0.48 (by CBDP-2 and CBDP-5), with an average of 0.31 . The mean values for $R p$ was 12.40 and ranged from 8.24 to 16.60 for primers CBDP-5 and CBDP-14, respectively. Furthermore, the highest and lowest values of $M I$ observed for primer CBDP-2 (5.31) and primer CBDP-15 (0.77), respectively, with an average of 2.63 (Table 1). The result of analysis of molecular variance (AMOVA) showed that more than $92 \%$ of the genetic diversity was partitioned within populations (two sets of genotypes), while the variation between the populations was $8 \%$ (Table 2).

This finding reveals that 90 percent of amplified fragments are informative for separation two genotype groups from each other, and suggesting that the majority of variation found is within genotype groups rather than between two groups. In addition, the results of population analysis showed that the diversity among populations (Gst) and gene flow $(\mathrm{Nm})$ were 0.054 and 8.70 , respectively (Table 2). Besides, a summary of the genetic diversity parameters of the two populations is shown in Table 3 . The percentage of poly- 
Table 1. CBDP primers and their amplification results generated in 50 durum wheats

\begin{tabular}{|c|c|c|c|c|c|c|c|}
\hline Primer & Sequence $\left(5^{\prime} \rightarrow 3^{\prime}\right)$ & $T A B$ & $N P B$ & $P P B$ & $P I C$ & $R p$ & MI \\
\hline CBDP-1 & TGAGCACGATCCAATAGC & 12 & 12 & 100 & 0.44 & 16.28 & 5.24 \\
\hline CBDP-2 & TGAGCACGATCCAATAAT & 11 & 11 & 100 & 0.48 & 13.04 & 5.31 \\
\hline CBDP-3 & TGAGCACGATCCAATACC & 9 & 9 & 100 & 0.20 & 15.92 & 1.84 \\
\hline CBDP-4 & TGAGCACGATCCAATAAG & 7 & 7 & 100 & 0.39 & 10.28 & 2.73 \\
\hline CBDP-5 & TGAGCACGATCCAATCTA & 9 & 9 & 100 & 0.48 & 8.24 & 4.36 \\
\hline CBDP-7 & TGAGCACGATCCAATCGA & 9 & 9 & 100 & 0.24 & 15.44 & 2.20 \\
\hline CBDP-9 & TGAGCACGATCCAATGAT & 8 & 8 & 100 & 0.43 & 10.92 & 3.47 \\
\hline CBDP-10 & TGAGCACGATCCAATGTT & 8 & 8 & 100 & 0.16 & 14.56 & 1.31 \\
\hline CBDP-12 & TGAGCACGATCCAATATA & 6 & 6 & 100 & 0.28 & 10.01 & 1.67 \\
\hline CBDP-13 & TGAGCACGATCCAATGAG & 5 & 5 & 100 & 0.27 & 8.36 & 1.37 \\
\hline CBDP-14 & TGAGCACGATCCAATGCG & 9 & 9 & 100 & 0.14 & 16.60 & 1.29 \\
\hline \multirow[t]{2}{*}{ CBDP-15 } & TGAGCACGATCCAATTGA & 5 & 5 & 100 & 0.15 & 9.16 & 0.77 \\
\hline & Mean & 8.17 & 8.17 & 100 & 0.31 & 12.40 & 2.63 \\
\hline
\end{tabular}

$T A B$ total amplified bands, $N P B$ number of polymorphic bands, $P P B$ percentage of polymorphic bands, $P I C$ polymorphism information content, $M I$ marker index, $R p$ resolving power.

Table 2. Analysis of molecular variance (AMOVA) based on CBDP markers for two populations of durum wheat

\begin{tabular}{|l|c|c|c|c|c|c|c|}
\hline \multicolumn{1}{|c|}{ Source } & $d f$ & SS & $M S$ & Est. Var & $\begin{array}{c}\text { Variation } \\
(\%)\end{array}$ & Gst & $N m$ \\
\hline Among pops & 1 & 12.97 & 12.97 & 0.86 & 8 & & \\
\hline Within pops & 48 & 491.04 & 10.23 & 10.23 & 92 & & \\
\hline Total & 49 & 504.01 & - & 11.09 & & 0.06 & 8.70 \\
\hline
\end{tabular}

$d f$ degree of freedom, $S S$ sum of squares, $M S$ mean of squares, Est. Var estimated variance components, Gst Inter-population differentiation; $\mathrm{Nm}$ Gene flow.

Table 3. Genetic variation features estimated using CBDP primers in the studied populations of durum wheat

\begin{tabular}{|l|c|c|}
\hline \multicolumn{1}{|c|}{ Parameters } & Breeding lines & Iranian landraces \\
\hline Observed number of alleles $(\mathrm{Na})$ & 1.82 & 1.91 \\
\hline Effective number of alleles $(\mathrm{Ne})$ & 1.50 & 1.61 \\
\hline Nei's genetic diversity $(\mathrm{He})$ & 0.29 & 0.35 \\
\hline Shannon's information index $(I)$ & 0.44 & 0.51 \\
\hline Percentage of polymorphic loci $(\mathrm{PPL})$ & 82.65 & 90.82 \\
\hline
\end{tabular}


morphic loci $(P P L)$ within populations was different, and the highest value observed in Iranian landraces group $(90.82 \%)$. Furthermore, landrace genotypes had the highest values for Shannon's index $(I=0.51)$, Nei's genetic diversity $(\mathrm{He}=0.35)$, the observed $(\mathrm{Na}=1.91)$ and effective $(\mathrm{Ne}=1.61)$ number of alleles. In order to explore relationships among the studied accessions, Jaccard's similarity coefficients was calculated according to the binary data matrix of amplified bands for the 50 durum wheat genotypes (coefficient matrix not shown). Pairwise genetic similarity ranged from 0.46 to 0.96 .

Accordingly, the highest genetic similarity was recorded between BL-22 and BL-26 while, the lowest similarity was between RL-30 and LR-37 individuals. These results recommend that CBDP markers are acceptable marker system for the determination of genetic diversity and relationships among durum wheat genotypes. The Fan-dendrogram also grouped the 50 individuals into three main clusters (Fig. 1), so that the clustering pattern nearly indicate clear pattern of clustering according to the population groups (breeding lines and landraces).

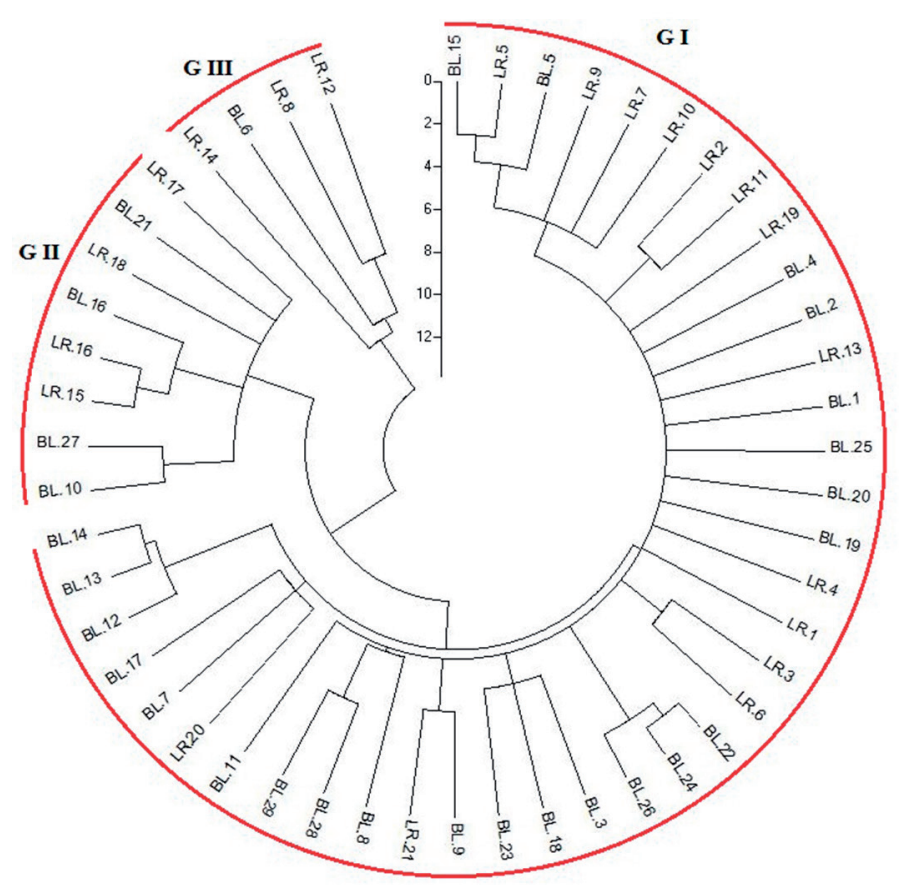

Figure 1. Jaccard's similarity coefficient-based Fan-dendrogram using CBDP data of 50 durum wheat genotypes. BL and LR indicated breeding line and landrace genotype, respectively (for explanation of individual codes, see Table S1) 


\section{Discussion}

The genetic diversity is the result of natural selection on the wild ancestor and human intermediation by ancient and modern farmers and breeders. The genomes of advanced lines, old cultivated landraces and wild relatives reflect the effects of these forces and provide insights into germplasm structural diversity. This issue is also of great practical importance for crop improvement because wild germplasm represents a rich potential source of useful under-exploited alleles or allele combinations (Jing et al. 2010). Molecular analysis across wheat germplasm, prior to collecting germplasm for conservation, would form a well-organized strategy for identifying unique populations with specific applicability for utilization in breeding aims. In recent years, progress in plant molecular tools has resulted in development of the gene-targeted and functional marker systems (Collard and Mackill 2009; Poczai et al. 2013). CAAT box-derived polymorphism (CBDP) technique is one of the novels gene-targeted marker system, which recently has been introduced for genetic analysis in many plant species. This technique activities CAAT box region, a different pattern of nucleotides with a consensus sequence, GGCCAATCT, situated upstream of the start codon of eukaryote genes that plays an important role during transcription, of promoters in plant genes (Singh et al. 2014).

There are several advantages recognizing this technique more appropriate than other molecular marker systems; (1) CBDP is a kind of gene-targeted molecular marker systems which characterized by simplicity and reproducibility. (2) This technique is based on PCR technology and it has many advantages such as low-cost, high polymorphic and extensive genetic information as well as it primers are universal in plants (Guo et al. 2012). (3) Compared to arbitrary markers, this marker is highly reproducible due to longer sequence primers. (4) CBDP technique is not plant species-specific thus, it can be employed to any plant groups or species (Singh et al. 2014). (5) In addition, this technique, as a gene-targeted marker, is more desirable for various applications in plant molecular genetics especially QTL mapping, since recombination levels between gene and marker or QTL are lower compared to RAPDs, SSRs or ISSRs (Andersen and Lubberstedt 2003).

In the present study, several CBDP primers were used to assess the level of genetic diversity among mini-core collection of durum wheat including two groups of genotypes, namely: Iranian landraces (as population 1) and breeding lines (as population 2). Our results revealed a high level of molecular variability among the studied genotypes. Similarly, the high levels of polymorphism has reported been in previous studies for durum wheat (Mardi et al. 2011; Alsaleh et al. 2016).

According to our results, CBDP primers showed high percentage of polymorphism bands $(P P B=100 \%)$. The marker informativeness parameters indicated good consent of discriminating power of these primers, suggesting a high efficiency of this DNA-marker technique to discover genetic diversity among durum wheat germplasm. Of these, polymorphism information content $(P I C)$ and resolving power $(R p)$ provide a degree of capability of marker technique, which help to determine the effectiveness and potential of the primers used in the fingerprinting process (Powell et al. 1996). Our results showed the $P I C$ values ranged from 0.14 to 0.48 , with an average of 0.31 . Among primers used, four primers had $P I C$ values above 0.40 , indicating that these primers are highly informative 
for determining polymorphism (Table 1). Although some primers showed the high value of $P I C$, the average value per primers was intermediate. A possible reason for this result is that the CBDP markers detect the polymorphism of the conserved regions surrounding the CAAT box regions, which is a consensus sequence upstream of the start codon (Singh et al. 2014).

Results of AMOVA (Table 2) revealed a higher distribution of genetic variation within durum populations $(92 \%)$ as compared to between populations $(8 \%)$. This is also supported by inter-population differentiation (Gst) value as a suitable parameter for the genetic variation. According to Nei (1978), our populations have been estimated to have the medium value of genetic differentiation coefficient (Gst) (0.06) (Table 2). In this respect, populations with Gst $>0.15$, between 0.05 and 0.15 and $<0.05$ have the high, medium and low levels of genetic differentiation, respectively (Hamrick et al. 1991). The number of migrants per generation $(\mathrm{Nm})$ is an estimated value from Gst to measure the gene flow, the higher its value, the less genetic differentiation among populations.

According to Wright's (1951) theory, if $N m>1$, it can inhibit the differentiation among populations due to genetic drift. On the other hand, if $N m<1$, local populations tend to differentiate. In the present study, the amount of $\mathrm{Nm}$ among studied populations was 8.70 (Table 2), indicating the possibility of minor gene flow between the studied populations of durum wheat. Indeed, the distribution of genetic variation between and within populations is a function of the rate of gene flow between them, and the extent of gene flow depends on the size and the degree of isolation of its populations as well as the movement seeds or pollens between populations (Dumolin-Lapegue et al. 1997).

The Nei's genetic diversity index $(\mathrm{He})$ and Shannon's index $(I)$ parameters have been identified as the most appreciate genetic variation parameters for indicate diversity and differentiation between and within the populations (Que et al. 2014). The higher values of these indices equale the greater the genetic diversity. In the present study, the extent of variability between $H$ and I indices revealed a high level of genetic diversity among the Iranian landraces (Table 3). These results indicate that the Iranian durum wheat landraces may be a good source of genetic diversity to be discovered in crosses with elite durum wheat germplasm. In agreement with our findings, Hamidi et al. (2014) using different marker techniques such as SSR, SCoT and CDDP markers, reported a high level of genetic variability among Iranian bread wheat landraces. Mardi et al. (2011) using SSAP, SSR and AFLP markers also demonstrated high diversity among the Iranian durum wheat germplasm.

According to the clustering pattern (Fig. 1), the genetic variation did not agree with the populations' viewpoint, as it can be seen that individuals related to breeding lines set or landraces group occur in different clusters, and on the contrary some of individuals from different groups clustered together. Furthermore, clustering dendrogram indicated that the Iranian landraces are divergent than breeding lines, which the main reason for this separation can be refer to its genetic background. This result can be support by AMOVA, which variation within genotype populations was higher than between populations (Table 2).

Breeding aims will advantage if focus is more on crosses between distant genotypes, or to enhance the diversity and identification of new genes and QTLs from landrace gen- 
otypes or other relative's crops for further wheat improvement. Iran is a main center of distribution and domestication of wheat with associated compositions of wheat ancestors as the richest wheat gene pool has been found in its' regions. To develop new cultivars and improve productivity of durum wheat, Iran has collaborated with the International Centre for Agricultural Research in the Dry Areas (ICARDA) for more than two decades. Hence, classifying and characterizing of wheat germplasm is an essential step in the selection and breeding of wheat. Genetic variation in the studied durum wheat collection based on CBDP analysis could be useful to select parents to be crossed for obtaining new appropriate populations intended for transgressive segregation of some important agronomic characters in the descendant populations. In conclusion, our results confirmed a high level of genetic diversity in the studied genotypes, especially Iranian landraces. Thus should be focus on collecting more landrace collections, as well as obtaining more genetic information for the purpose of improvement and development of new populations. In addition, the results revealed that the CBDP technique was a highly reproducible, efficient and powerful tools to assess genetic diversity among durum wheat genotypes. More importantly, we indicated that either the CBDP markers could be utilized individually or in combination with other markers to accurate evaluate genetic variation of wheat germplasm and to obtain reliable information about population structure. Thus, the genetic analyses using this marker system would be more useful for crop improvement programs, such as genotype identification, assessing genetic diversity, construction of linkage maps and QTL mapping.

\section{Acknowledgement}

The authors are grateful to Kermanshah Branch, Islamic Azad University for hosting the lab facilities.

\section{References}

Alikhani, L., Rahmani, M.S., Shabanian, N., Badakhshan, H., Khadivi-Khub, A. 2014. Genetic variability and structure of Quercus brantii assessed by ISSR, IRAP and SCoT markers. Gene 552:176-183.

Alsaleh, A., Shehzad Baloch, F., Nachit, M., Ozkan, H. 2016. Phenotypic and genotypic intra-diversity among Anatolian durum wheat "Kunduru" landraces. Biochem. Syst. Ecol. 65:9-16.

Andersen, J.R., Lubberstedt, T. 2003. Functional markers in plants. Trends Plant. Sci. 8:554-560.

Benoist, C., Ohare, K., Breathnach, R., Chambon, P. 1980. The ovalbumin gene-sequence of putative control regions. Nucleic Acids Res. 8:127-142.

Collard, B.C.Y., Mackill, D.J. 2009. Conserved DNA-derived polymorphism (CDDP): a simple and novel method for generating DNA markers in plants. Plant. Mol. Biol. Report. 27:558-562.

Doyle, J.J., Doyle, J.L. 1987. A rapid DNA isolation procedure for small quantities of fresh leaf tissue. Phytochemical. Bulletin 19:11-15.

Dumolin-Lapegue, S., Demesure, B., Fineschi, S., Le Corre, V., Petit, R.J. 1997. Phylogeographic structure of white oaks throughout the European continent. Genetics 146:1475-1487.

Etminan, A., Pour-Aboughadareh, A., Mohammadi, R., Ahmadi-Rad, A., Noori, A., Mahdavian, Z., Moradi, Z. 2016. Applicability of start codon targeted (SCoT) and inter-simple sequence repeat (ISSR) markers for genetic diversity analysis in durum wheat genotypes. Biotechnol. Biotec. Eq. 30:1075-1081. 
Guo, D.L., Zhang, J.Y., Liu, C.H. 2012. Genetic diversity in some grape varieties revealed by SCoT analyses. Mol. Biol. Rep. 39:5307-5313.

Hamidi, H., Talebi, R., Keshavarz, F. 2014. Comparative efficiency of functional gene-based markers, start codon targeted polymorphism (SCoT) and conserved DNA-derived polymorphism (CDDP) with ISSR markers for diagnostic fingerprinting in wheat (Triticum aestivum L.). Cereal. Res. Commun. 42:558-567.

Hamrick, J.L., Godt, M.J.W., Murawski, D.A., Loveless, M.D. 1991. Correlation between species traits and allozyme diversity: implications for conservation biology. In: Falk, D.A., Holsinger, K.E. (eds), Genetic and Conservation of Rare Plants. Oxford University Press. New York, USA. pp. 75-86.

Jaccard, P. 1908. New research on the floral distribution. Sci. Naturelles 44:223-270.

Jing, R., Vershinin, A., Grzebyta, J., Shaw, P., Smykal, P., Marshall, D., Ambrose, M.H., Ellis, T.H.N., Flavell, A.J. 2010. The genetic diversity and evolution of field pea (Pisum) studied by high throughput retrotransposon based insertion polymorphism (RBIP) marker analysis. BMC Evol. Biol. 10:44.

Mardi, M., Naghavi, M.R., Pirseyedi, S.M., Kazemi Alamooti, M., Rashidi Monfared, S., Ahkami, A.H., Omidbakhsh, M.A., Alavi, N.S., Salehi Shanjani, P., Katsiotis, A. 2011. Comparative assessment of SSAP, AFLP and SSR markers for evaluation of genetic diversity of durum wheat (Triticum turgidum L. var. durum). J. Agr. Sci. Tech. 13:905-920.

Nei, M. 1978. Estimation of average heterozygosity and genetic distance from a small number of individuals. Genetic 89:583-590.

Peakall, R., Smouse, P.E. 2006. GENALEX 6: genetic analysis in excel. Population genetic software for teaching and research. Mol. Ecol. Notes 6:288-295.

Poczai, P., Varga, I., Laos, M., Cseh, A., Bell, N., Valkonen, J.P. Hyvonen, J. 2013. Advances in plant genetargeted and functional markers: a review. Plant. Methods. 9:1-31.

Pour-Aboughadareh, A., Mohmoudi, M., Ahmadi, J., Moghaddam, M., Mehrabi, A.A., Alavikia, S.S. 2017. Agro-morphological and molecular variability in Triticum boeoticum accessions from Zagros Mountains, Iran. Genet. Resour. Crop. Evol. 64:545-556.

Poursiahbidi, M., Pour-Aboughadareh, A., Tahmasebi, G., Seyedi, A, Jasemi, M. 2012. Factor analysis of agromorphological characters in wheat (Triticum durum Def.) lines. Int. J. Agric. Crop. Sci. 23:1758-1762.

Poursiahbidi, M., Pour-Aboughadareh, A., Tahmasebi, G., Teymoori, M., Jasemi, M. 2013. Evaluation of genetic diversity and interrelationships of agro-morphological characters in durum wheat (Triticum durum desf.) lines using multivariate analysis. Intl. J. Agric. Res. Rev. 3:184-194.

Powell, W., Morgante, M., Andre, C., Hanafey, M.M., Vogel, J., Tingey, S., Rafalski, A. 1996. The comparison of RFLP, RAPD, AFLP and SSR (microsatellite) markers for germplasm analysis. Mol. Breed. 2:225-238.

Que, Y., Pan, Y., Lu, Y., Cui, Y., Yuting, Y., Ning, H., Xu, L. 2014. Genetic analysis of diversity within a Chinese local sugarcane germplasm based on Start Codon Targeted Polymorphism. Bio. Med. Res. Int. ID 468375:1-10.

Singh, A.K., Rana, M.K., Singh, S., Kumar, S., Kumar, R., Singh, R. 2014. CAAT box-derived polymorphism (CBDP): a novel promoter-targeted molecular marker for plants. J. Plant. Biochem. Biotechnol. 23:175-183.

Tamura, K., Peterson, D., Peterson, N., Stecher, G., Nei, M., Kumar, S. 2011. MEGA5: molecular evolutionary genetics analysis using maximum likelihood, evolutionary distance, and maximum parsimony methods. Mol. Biol. Evol. 28:2731-2739.

Wright, S. 1951. The genetical structure of populations. Ann. Eugen. 15:323-354.

Yeh, F., Yang, R., Boyle, T. 1997. POPGENE, the user friendly shareware for population genetic analysis. Molecular Biology and Biotechnology Center. University of Alberta, Edmonton.

\section{Electronic Supplementary Material (ESM)}

Electronic Supplementary Material (ESM) associated with this article can be found at the website of CRC at http://www.akademiai.com/content/120427/

Electronic Supplementary Table S1. Passport of the studied 50 durum wheat genotypes 